\title{
Status Epilepticus Due to Organophosphate Poisoning in an Infant
}

\author{
Parmar $\mathrm{PN}^{1}$
}

\begin{abstract}
We report a case of a three months old female presented with status epilepticus who was diagnosed as organophosphate (OP) poisoning. She was from rural area with agricultural background. Typical clinical features and history of exposure to organophosphate compound were absent. The infant was successfully treated with atropine, pralidoxime and supportive management. Safety and efficacy data of pralidoxime in infants are limited in literature and was found safe in our case.
\end{abstract}

Key words: Infant, Oximes, Status Epilepticus

\section{Introduction}

$\mathrm{U}$ nintentional organophosphate (OP) poisoning in children is common ${ }^{1}$. Exposure can occur via skin and mucous membranes, inhalation or ingestion.

OP poisoning is usually diagnosed by characteristic clinical features, low serum or red blood cell cholinesterase level and a history of exposure to a known OP compound ${ }^{2}$. Clinical features of organophosphate poisoning in children are different from those in adults $^{1}$ and history of exposure may not be found in some children ${ }^{3}$. Diagnosis of OP poisoning may be missed in such cases. Treatment of OP poisoning includes supportive therapy and atropine, oximes, benzodiazepines, gastrointestinal decontamination and experimental therapies ${ }^{2}$. Usefulness and safety of oximes in acute OP poisoning have been questioned recently ${ }^{4}$ and data for paediatric patients are even fewer.

We report a three month old female presenting as status epilepticus who developed pinpoint pupils and excessive bronchial secretions after 12 hours of admission. She was diagnosed OP poisoning. Source and route of exposure was not known after detailed inquiry of parents. The patient was treated successfully with supportive treatment and intravenous atropine and pralidoxime.

\section{The Case}

A three month old female was presented with sudden onset of excessive crying for three hours and generalized tonic clonic seizures for last one hour. There was no history of fever, feeding difficulty,
'Dr. Pareen N Parmar, MBBS, MD, (Paediatrics), DAA (CMC, Vellore - International Asthma Services), CFMT (CSMT, Chennai), CRM (IIPH-G, Ahmedabad), Consultant Paediatric Allergy-Asthma Specialist, Music Therapy Consultant Rajkot, Gujarat (India)

Address for correspondence:

Dr. Parin N Parmar

E-mail: parinnparmar@gmail.com

\section{How to cite}

Parmar PN. Status Epilepticus Due to Organophosphate Poisoning in an Infant. J Nepal Paediatr Soc 2014;34(3):247-249.

doi: http://dx.doi.org/10.3126/jnps.v34i3.10497

This work is licensed under a Creative Commons Attribution 3.0 License.

\section{(c) (7)}

respiratory or gastrointestinal complaints, bleeding tendency or trauma. Her father was a farmer and was residing in a village. She was born of a non-consanguineous marriage with uneventful birth history and her birth weight was $2.5 \mathrm{~kg}$. She was exclusively breastfed and her growth and development were normal. She was not on any medication. Her weight was 5.6 $\mathrm{kg}$ and head circumference was $39 \mathrm{~cm}$. On examination, she was afebrile, pulse rate was $132 /$ minute, and respiratory rate was $42 /$ minute. On systemic examination, both pupils were constricted and reactive to light and rest of the examination was uninformative. After taking relevant samples, she was treated with intravenous calcium gluconate, dextrose, antibiotics, phytomenadion, loading dose of phenobarbitone and maintenance intravenous fluids. 
Investigations on the first day of admission were as follows: Hemoglobin $8.7 \mathrm{gm} / \mathrm{dL}$, total white blood cell count $16000 / \mathrm{mm}^{3}$ with $58 \%$ neutrophils and $40 \%$ lymphocytes, platelet count $8.7 \mathrm{lacs} / \mathrm{mm}^{3}$, plasma glucose $157 \mathrm{mg} / \mathrm{dL}$, prothrombin time 15.5 seconds with INR 1.15, activated partial thromboplastin time 26.7 seconds, serum ionized calcium $1.15 \mathrm{mmol} / \mathrm{L}$. Examination of cerebrospinal fluid showed 3-5 lymphocytes $/ \mathrm{mm}^{3}$ and culture was negative for bacterial organism.

During the hospital stay, the patient developed increasing tachypnoea with respiratory secretions. At 12 hours of admission, pulse rate was 146/minute, respiratory rate was $64 /$ minute with bilateral secretory sounds in lung fields. There was no recurrence of seizure but the patient did not regain consciousness. Both pupils were pinpoint with rest of the central nervous system examination uninformative. Chest $X$-ray was normal. Organophosphate poisoning was suspected and serum cholinesterase level by enzymatic method was $99 \mathrm{U} / \mathrm{L}$ (normal range: 5900-12220 U/L) confirming diagnosis of organophosphate poisoning.

In addition to supportive management, the patient was treated with intravenous atropine and pralidoxime chloride. Atropine was given as 0.05 $\mathrm{mg} / \mathrm{kg} /$ dose intravenously every 15 minutes until clearing of bronchial secretions and then as needed. Pralidoxime was given as 2 intermittent doses of 50 $\mathrm{mg} / \mathrm{kg}$ at interval of 12 hours as intravenous infusion over 30 minutes each. The patient showed clinical improvement within 90 minutes of initiation of specific therapy and atropine was safely tapered after 48 hours. There was no untoward reaction. The source of organophosphate was not found after detailed history.

\section{Discussion}

Common presenting symptoms and signs of OP poisoning in children are pinpoint pupils, excessive respiratory secretions, diarrhea, vomiting, altered sensorium, respiratory failure, 'garlic odor' from body, shock, apathy, muscle weakness, etc ${ }^{3}$.

As our patient presented with excessive crying and status epilepticus only, meningitis, metabolic abnormalities, and intracranial hemorrhage were suspected initially. Presence of constricted pupils on admission was not sufficient to suspect OP poisoning. It was suspected after 12 hours of admission when patient developed pinpoint pupils and excessive bronchial secretions. Although diarrhea is an important feature in OP poisoning in children less than 5 years of $a^{a g}{ }^{5}$, our patient did not develop diarrhea during the course.

No source was found after making detailed enquiry for possible exposures. No insecticide or any spray was used inside the house or in vicinity of house in last week. There was no history of any ingestion or application over skin and mucous membranes. No soap or shampoo ${ }^{6}$ was used for bathing.

Children of agricultural workers are at high risk for development of both acute and chronic neurological symptoms ${ }^{7}$. The father being a farmer and residence in rural area were important clues.

Unusual presentations of OP poisoning such as presentation as diabetic ketoacidosis ${ }^{8}$, transplacentally acquired OP poisoning in a newborn ${ }^{9}$, OP poisoning complicating as extrapyramidal parkinsonism ${ }^{10}$, etc have been reported in literature. In our case, age of the patient and her clinical presentation were unique.

There are no well-controlled clinical trials to establish effectiveness and safety of pralidoxime chloride in infants is as OP poisoning in this age group is not common. Our patient did not develop any side effect with use of intermittent infusion dosing of pralidoxime.

Major limitation in our case report is that we do not know whether it was a carbamate poisoning. So we cannot comment about effectiveness of pralidoxaime in our case.

To summarize, we report a three month old patient from agricultural family background who presented with status epilepticus. She was diagnosed OP poisoning after 12 hours of admission due to presence of pinpoint pupils and excessive bronchial secretions. Source and mode of exposure was not known. She improved with supportive therapy with intravenous atropine and intermittent infusion dosing of pralidoxime. Pralidoxime was safe in the infant.

\section{Conclusion}

This report emphasizes importance of high index of suspicion to diagnose OP poisoning in infants, especially those coming from agricultural or rural areas. Presence of pinpoint pupils and bronchial secretions are a strong clue. History of exposure may not be present. Intermittent infusion dosing of pralidoxime was safe in our case. 


\section{References}

1. Levy-Khademi F, Tenenbaum AN, Wexler ID, Amitai Y. Unintentional organophosphate intoxication in children. Pediatr Emerg Care 2007;23(10):716-8.

2. Kumar SV, Fareedullah Md, Sudhakar Y, Venkateswarlu B, Kumar EA. Current review on organophosphorus poisoning. Archives of Applied Science Research 2010; 2(4): 199-215.

3. Dippenaar R, Diedericks RJ. Paediatric organophosphate poisoning - a rural hospital experience. S Afr Med J 2005;95:678-81.

4. Samuel J, Peter JV, Thomas K, Jeyaseelan L, Cherian AM. Evaluation of two treatment regimens of pralidoxime (1gm single bolus dose vs. 12gm infusion) in the management of organophosphorus poisoning. J Assoc Physic India 1996;44:529-531.

5. Bond GR, Pieche S, Sonicki Z, Gamaluddin H, El Guindi $M$, Sakr M, El Seddawy A, Abouzaid M, Youssef A; WHO EMRO Pediatric Insecticide Study Group. A clinical decision aid for triage of children younger than 5 years and with organophosphate or carbamate insecticide exposure in developing countries. Ann Emerg Med 2008;52(6):617-22.
Doi: $\quad 10.1016 /$ j.annemergmed.2008.03.026.Epub 2008 Jun 16.

6. Sadaka $\mathrm{Y}$, Broides $\mathrm{A}$, Tzion RL, Lifshitz $\mathrm{M}$. Organophosphate acetylcholine esterase inhibitor poisoning from a home-made shampoo. J Emerg Trauma Surg 2011;4(3):433-34.

7. Rastogi SK, Tripathi S, Ravishanker D. A study of neurologic symptoms on exposure to organophosphate pesticides in the children of agricultural workers. Indian J Occupational Environ Med 2010;14(2):54-7. Doi:10.4103/00195278.72242.

8. Kumar KJ, Nayak N. Organophosphorus poisoning presenting as diabetic ketoacidosis. Indian Pediatr 2011;48(1):74.

9. Jajoo M, Saxena S, Pandey M. Transplacentally acquired organophosphorus poisoning in a newborn: care report. Ann Trop Paediatr 2010;30(2):137-9. Doi: 10.1179/14654281x12703 902516202.

10. Shahar E, Bentur Y, Bar-Joseph G, Cahana A, Hershman E. Extrapyramidal parkinsonism complicating acute organophosphate insecticide poisoning. Pediatr Neurol 2005;33(5):378-82. 